\title{
The L76V mutation in HIV-1 protease is potentially associated with hypersusceptibility to protease inhibitors Atazanavir and Saquinavir: is there a clinical advantage?
}

Frank Wiesmann ${ }^{1 *}$, Jan Vachta', Robert Ehret ${ }^{1}$, Hauke Walter ${ }^{2}$, Rolf Kaiser ${ }^{3}$, Martin Stürmer ${ }^{4}$, André Tappe ${ }^{5}$, Martin Däumer ${ }^{6}$, Thomas Berg ${ }^{7}$, Gudrun Naeth', Patrick Braun ${ }^{1}$, Heribert Knechten ${ }^{1}$

\begin{abstract}
Background: Although being considered as a rarely observed HIV-1 protease mutation in clinical isolates, the L76V-prevalence increased 1998-2008 in some European countries most likely due to the approval of Lopinavir, Amprenavir and Darunavir which can select L76V. Beside an enhancement of resistance, L76V is also discussed to confer hypersusceptibility to the drugs Atazanavir and Saquinavir which might enable new treatment strategies by trying to take advantage of particular mutations.
\end{abstract}

Results: Based on a cohort of 47 L76V-positive patients, we examined if there might exist a clinical advantage for L76Vpositive patients concerning long-term success of PI-containing regimens in patients with limited therapy options. Genotypic- and phenotypic HIV-resistance tests from 47 mostly multi-resistant, L76V-positive patients throughout Germany were accomplished retrospectively 1999-2009. Five genotype-based drug-susceptibility predictions received from online interpretation-tools for Atazanavir, Saquinavir, Amprenavir and Lopinavir, were compared to phenotype-based predictions that were determined by using a recombinant virus assay along with a Virtual Phenotype ${ }^{\mathrm{TM}}(\mathrm{Virco})$. The clinical outcome of the L76V-adapted follow-up therapy was determined by monitoring viral load for 96 weeks.

Conclusions: In this analysis, the mostly used interpretation systems overestimated the L76V-mutation concerning Atazanavir- and SQV resistance. In fact, a clear benefit in drug susceptibility for these drugs was observed in phenotype analysis after establishment of L76V. More importantly, long-term therapy success was significantly higher in patients receiving Atazanavir and/or Saquinavir plus one L76V-selecting drug compared to patients without L76V-selecting agents $(p=0.002)$.

In case of L76V-occurrence ATV and/or SQV may represent encouraging options for patients in deep salvage situations.

\section{Background}

The reduced susceptibility to certain antiretrovirals is often accompanied with a gradual loss of viral fitness, indicating that mutations with high fitness costs are less able to persist in the absence of drug pressure [1]. There have been recent reports about HIV strains with increased susceptibility to particular drugs when certain

\footnotetext{
* Correspondence: f.wiesmann@googlemail.com

${ }^{1}$ PZB Aachen, HIV\&Hepatitis Research Group, Blondelstr., 52062 Aachen, Germany

Full list of author information is available at the end of the article
}

mutation patterns had developed under antiretroviral treatment [2-5]. This biological attribute enables new putative strategies for future treatment of HIV-infected patients with abundant resistance mutations by trying to take advantage of particular mutations [6].

As example, M184V/I, the most prevalent NRTImutations selected under 3TC or FTC in the reverse transcriptase, do for instance revert partially the effect of thymidine-analogue mutation- (TAM) on resistance [7]. K65R and L74V are further mutations which can confer hypersusceptibility or resensitization to AZT [8].

\section{Ciomed Central}


Beside these specific mutations in the reverse transcriptase, there are also reports about resensitizing mutations affecting the protease gene $[9,10]$.

\section{Objectives}

This article reports about possible clinical advantages of a valine substitution, instead of leucine, at position 76 in the HIV-1 protease. This mutation generally disappears quickly in replicating viruses in absence of selection pressure mediated by LPV, APV or DRV treatment. Thus, for deep salvage therapy situations in patients with strongly limited therapy options, it might be of advantage to maintain these drugs in treatment regimens to preserve $\mathrm{L} 76 \mathrm{~V}$ in the current replicating virus in combination with a "resensitized" drug ATV or SQV.

\section{Results}

Patients with protease gene mutation L76V show increased susceptibility for Atazanavir and Saquinavir At first, the impact of L76V on ATV- and SQVresistance characteristics was assessed before and after establishment of the mutation. Due to the manifestation of the L76V mutation as well as other minor mutations at resistance-relevant sites in the course of treatment, genotype-based interpretation tools predicted intermediate or mostly complete resistance against all PIs including ATV and SQV and the majority of NRTIs and NNRTIs resulting in an active drug score (ADS) of $\leq$ 1.0 for the failing regimen (Figure 1). Interestingly, in phenotypic analysis, the resistance factor (RF) for ATV and SQV remained at full susceptibility in both patients and even decreased for SQV from 31 to 1.1 (Figure $1 \mathrm{~A}$ ) and 1.1 to 0.6 (Figure $1 \mathrm{~B}$ ) and for ATV from 62 to 2.8 and 4.3 to 0.9 , respectively.

In a further aspect, genotypic and phenotypic resistance data of 10 patients, all L76V positive, was assessed in order to analyze if these observed resensitizing effects represent ubiquitous drug resistance patterns. Figure 2 supports this hypothesis on a variety of other patients harbouring HIV populations with L76V mutation. The accuracy and concordance of predicted genotype-based interpretations were compared with obtained phenotypic

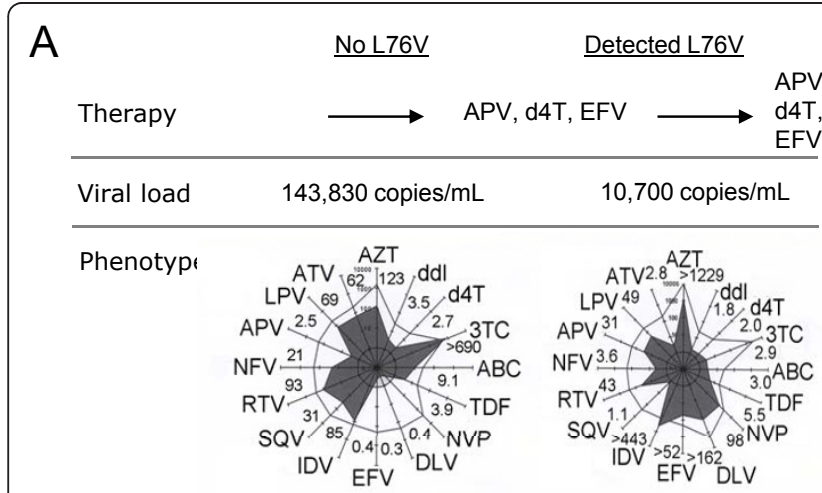

日 Max. Resistance

Resistance Factor (present sample)

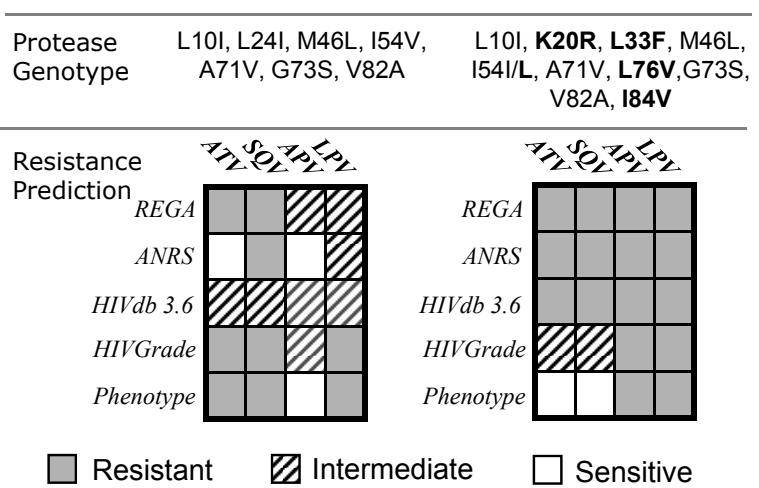

B

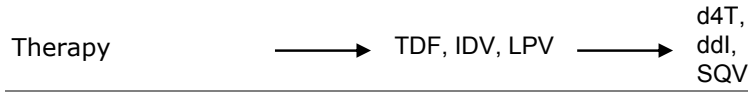

Viral load 11,488 copies $/ \mathrm{mL}$ 859,000 copies $/ \mathrm{mL}$

$\boxminus$ Max. Resistance $\square$ Resistance Factor (present sample)

Protease
Genotype $\quad$ L10I, M46I, I54V, V82F $\begin{gathered}\text { L10I, K20R, M46I, I54V, } \\ \text { A71V,L76V,V82F }\end{gathered}$

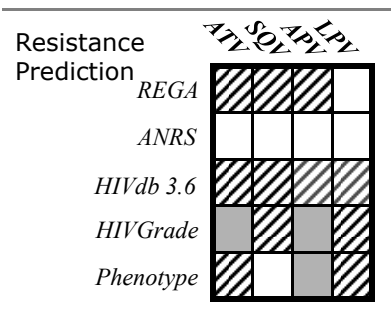

Resistant $\bigotimes$ Intermediate

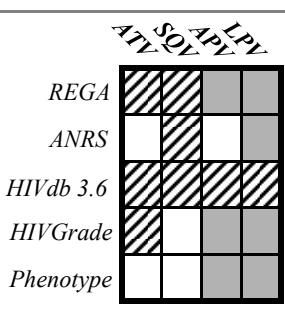

Sensitive

Figure 1 Resensitizing effects of the L76V mutation are visible in phenotype results: Phenotypic resistance analysis before and after manifestation of L76V in two representative patients ( $A+B)$. Although additional mutations developed in the progress of therapy (bold characters) the resistance factor for ATV and SQV decreased below the cut-off for full susceptibility in both patients compared to analyses one year before. Antiretroviral drugs are illustrated with corresponding resistance factors (cut-off: 0-3.5 = sensitive 3.6-9.5 (29 for LPV) = intermediate; $>9.0$ (29 for LPV) = resistant). Genotypic resistance interpretations derived from five common online tools showed considerable discrepancies in weighting of ATV and SQV resistance levels compared to each other and to phenotypic results (grey and white colour). A) One patient with failing APV containing therapy after week 72. B) Another patient with a failing IDV/LPV treatment before start of SQV containing therapy. 
resistance levels from recombinant virus assays and virtual phenotype analysis.

Despite a general concordance in genotype- and phenotype-based resistance predictions for LPV and APV, there were wide discrepancies in the weighting of resistance for ATV and SQV, mostly overestimation of resistance in genotype-based predictions (Figure 2). However, most phenotypic resistance interpretations uncovered full susceptibility for the drugs ATV and SQV. In most cases L76V appeared to be associated with a variety of other resistance relevant protease mutations without effecting the resensitizing effect. However, in particular, the copresence of the protease mutation L90 M was notably associated with high ATV and SQV resistance factors (Figure 2; \#4, \#26, \#21).
Clinical outcome and follow-up in patients with L76Vadapted therapy

Considering the effect of L76V on susceptibility for ATV and SQV, the big question was obviously, how this mutation might affect the therapeutic option and strategy for patients with a narrow margin of remaining active drugs. A considerable issue remained to generalize data from a small cohort of patients with diverse optimized backbone therapies. Thus, this work focused on the amount of active drugs in the treatment of each patient. Table 1 shows the L76V-adjusted follow-up therapies that were administered after resistance prediction results.

Sufficient virus suppression below the detection limit was initially observed in 50\% of group A (ATV and/or

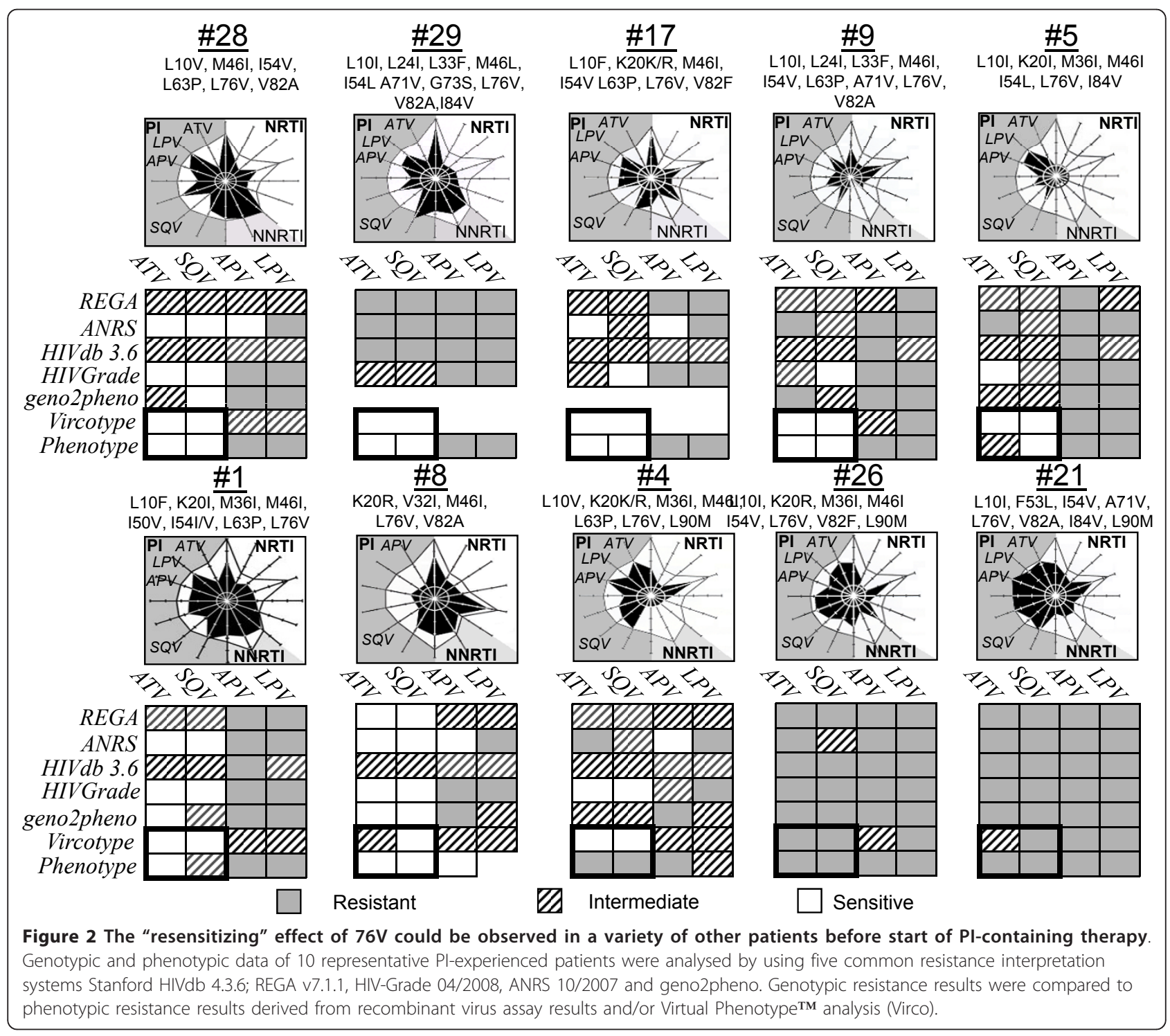


SQV without L76V-selecting drug) and $67 \%$ of group B (ATV and/or SQV plus L76V-selecting drug LPV or APV) within the first weeks of follow-up therapy. Despite similar response rates at first, a sustained therapy success with virus suppression still below 50 copies/ $\mathrm{mL}$ at week 96 and longer was predominantly achieved in group B patients where the selection pressure on L76V was constantly maintained by the drugs LPV or APV (Table 1, lower rows). While $66.7 \%$ of group B patients remained under detection levels at week 96 , there was a significantly lower success rate in group A patients with $16.7 \%$ remaining $<50$ copies $/ \mathrm{mL}$ in perprotocol analysis $\left(\mathrm{p}=0.002 ; \chi^{2}\right.$ test) (Table 2 and Figure 3). Patients of group $\mathrm{C}$ did not show any virus suppression below detection limit.

Interestingly, despite a successful virus suppression $<50$ copies/mL, the majority of group B therapies were expected to have an ADS below 2.0 after genotypic resistance predictions, indicating a very likely event of therapy failure (bold numbers in square brackets) (Table 1).

Most of the cases where therapies were predicted to have active drug scores of $\geq 2.0$ turned out to be successful. Only few experienced virological failure despite an ADS of more than 2.0 (indicated in round brackets).

A major question remained obviously, why patients of group A display earlier therapy failures than patients of group B. After two years of follow-up therapy, only one patient of group A, who additionally received a fusioninhibitor containing treatment, showed a viral load still below 50 copies $/ \mathrm{mL}$. The median viral load increased after 24 weeks of follow-up in group A (Figure 3). More interestingly, due to a loss of selection pressure on the L76V mutation, it was then undetectable in those patients who failed therapy, resulting in a decrease of the ADS below <2.0 (Table 3). While L76V was undetectable in patients where no $\mathrm{L} 76 \mathrm{~V}$-selecting drug was applied, it persisted in group B and group $C$ where selection pressure on mutation $\mathrm{L} 76 \mathrm{~V}$ was maintained (Table 3). In these patients the therapy failure had other reasons (e.g. acquisition of L90M).

These results additionally indicate benefits for patients with L76V-selecting drugs in combination with L76V"resensitized" drugs. A major issue remains the establishment of additional protease gene mutations i.e. L90 $\mathrm{M}$ and further compensatory changes over the time (Figure 2; \#4, \#21, \#26), making it crucial to suppress the virus completely and monitor viral load in close intervals.

\section{Discussion}

Little is known about the impact of drug-resensitizing mutations on antiretroviral therapy. Most works mainly describe the effects of resistance mutations on reductions in drug susceptibility. However, selective pressure of drug therapy may also lead to shifts in the quasispecies distribution and fitness of those mutants with decreased sensitivity to the respective antiretrovirals $[11,12]$. This loss in replication fitness may be even larger for a multi-drug resistant virus and might lead to a better starting point for particular antiretroviral regimens [13]. Nevertheless, it is not always applicable that the acquisition of drug-resistance mutations inevitably result in loss of viral fitness. Even in case a loss is apparent, the virus may select compensatory changes over time $[12,14]$. This may explain why current treatment guidelines still advocate a switch to antiretroviral treatment regimens following the emergence of drug resistance mutations and possibly prior to selection of compensatory changes [11]. In summary, drug hypersusceptibility mutations which reduce viral fitness are difficult to maintain in the predominant virus population in multiple pretreated patient.

In this article we provide insights into a possibility how to maintain efficient selection pressure on the protease mutation at position L76V by combining one drug, which selects L76V (in this case LPV, APV, DRV) and another drug which gains efficiency when L76V develops. This article reports about a significant clinical benefit of the protease mutation L76V on drug susceptibility to ATV and SQV due to resensitizing effects in multi-resistant patients resulting in a significantly higher long-term therapy success. These results may be in line with explanations from molecular dynamics- and free energy studies recently reported by Alcaro et al. who found that in the presence of the L76V substitution, ATV reveals a more productive binding affinity, in agreement with hypersusceptibily data [15].

\section{Conclusion}

The strategy of combining mutation-selecting drugs with "resensitized" drugs has already been discussed for the reverse transcriptase mutation M184V in NRTIcontaining therapies $[6,13,16]$ and has also been shown to be an adequate option for a couple of other mutations including the mutation N88 S [10].

Despite initially adequate therapy response rates in $50 \%$ (group A) - $67.7 \%$ (group B) of cases, it remains a major issue that virological failure under these therapies often occur due to compensatory changes in the virus genotype over the time mostly due to an additional establishment of further mutations in the respective gene [12,14]. As shown in Table 3, failure of therapy in L76V-positive patients with ATV and/or SQV containing therapy was noticeable associated with an additional establishment of the protease gene mutation at position L90 $\mathrm{M}$ which resulted in resistance against all available PIs [17]. Six of eight patients who received a second genotypic resistance test following 


\begin{tabular}{|c|c|c|c|c|c|c|c|c|c|c|c|c|c|c|c|c|c|c|c|c|c|c|c|c|c|c|c|c|}
\hline \multirow[b]{2}{*}{ Pat-ID } & \multicolumn{10}{|c|}{ GROUP A } & \multicolumn{18}{|c|}{ GROUP B } \\
\hline & $\# 1$ & \#2 & $\# 4$ & $\# 12$ & $\# 14$ & $\# 17$ & $\# 18$ & $\# 21$ & \#22 & \#46 & \#3 & $\# 5$ & \#8 & $\# 9$ & $\# 10$ & $\# 11$ & $\# 16$ & $\# 19$ & $\# 24$ & $\# 25$ & $\# 26$ & $\# 27$ & $\# 31$ & $\# 33$ & $\# 37$ & $\# 39$ & $\# 40$ & $\# 41$ \\
\hline \multirow[t]{6}{*}{ Therapy } & ATV & ATV & ATV & $A T V / r$ & SQV & $d d l$ & ATV & ATV & ATV/r & SQVI & $L P V / r$ & $L P V / r$ & $L P V / r$ & $L P V / r$ & $\angle P V$ & $L P V / r$ & $\mathrm{LPV} / \mathrm{r}$ & $L P V / r$ & APV & APV & $A P V / r$ & $L P V / r$ & $L P V / r$ & $L P V / r$ & $L P V / r$ & $L P V / r$ & $L P V / r$ & $L P V / r$ \\
\hline & SQV & SQV & SQV & SQV & $T P V / r$ & $d 4 T$ & SQV & EFV & SQV & r & SQV & ATV & SQV & SQV & ATV & ATV & SQV & SQV & SQV & SQV & SQV & SQV & SQV & SQV & SQV & SQV & SQV & ATV \\
\hline & $R T V$ & RTV & $R T V$ & $3 T C$ & $3 T C$ & SQV & $3 T C$ & TDF & $A Z T$ & TDF & $3 T C$ & AZT & $d 4 T$ & ENF & FTC & EFV & & & TDF & $\mathrm{ddC}$ & & FTC & $3 T C$ & $A Z T$ & $d d l$ & TDF & TDF & TDF \\
\hline & FTC & $3 T C$ & & T2O & $d d l$ & & T2O & AZT & $3 T C$ & FTC & $d 4 T$ & 3TC & & & TDF & & & & & $\mathrm{d} 4 \mathrm{~T}$ & & TDF & ETR & $3 T C$ & $3 T C$ & & MVC & $A Z T$ \\
\hline & & & & & & & & $3 T C$ & TDF & & TDF & & & & & & & & & & & & & TDF & & & & $A B C$ \\
\hline & & & & & & & & MVC & & & & & & & & & & & & & & & & & & & & $3 T C$ \\
\hline Rev.- & $41 \mathrm{~L}$ & $41 \mathrm{~L}$ & $41 \mathrm{~L}$ & $41 \mathrm{~L}$ & $41 \mathrm{~L}$ & $67 \mathrm{~N}$ & $41 \mathrm{~L}$ & $41 \mathrm{~L}$ & $41 \mathrm{~L}$ & n.d. & $41 \mathrm{~L}$ & $65 R$ & $67 \mathrm{~N}$ & $41 \mathrm{~L}$ & $41 \mathrm{~L}$ & $41 \mathrm{~L}$ & $41 \mathrm{~L}$ & no & no & no & $67 \mathrm{~N}$ & $41 \mathrm{~L}$ & $41 \mathrm{~L}$ & $41 \mathrm{~L}$ & $41 \mathrm{~L}$ & $67 \mathrm{~N}$ & $67 \mathrm{~N}$ & $41 \mathrm{~L}$ \\
\hline Transcriptase & $44 \mathrm{D}$ & $67 \mathrm{ss}$ & 1181 & $44 \mathrm{~A}$ & $67 \mathrm{~N}$ & $70 R$ & $67 \mathrm{~N}$ & $67 \mathrm{~N}$ & $44 \mathrm{D}$ & & $67 s 5$ & $70 R$ & $184 \mathrm{~V}$ & $44 \mathrm{D}$ & $44 \mathrm{D}$ & $67 \mathrm{~N}$ & $44 \mathrm{D}$ & data & data & data & $70 R$ & $67 \mathrm{~N}$ & $44 \mathrm{~A}$ & $74 \mathrm{~V}$ & $44 \mathrm{D}$ & 70R & $70 R$ & $67 \mathrm{~N}$ \\
\hline \multirow[t]{8}{*}{ mutations } & $67 \mathrm{~N}$ & 695 & $184 \mathrm{~V}$ & $67 \mathrm{~N}$ & $69 \mathrm{D}$ & 103 NS & $69 \mathrm{E}$ & $74 \mathrm{~V}$ & $67 \mathrm{~N}$ & & 695 & $103 \mathrm{~N}$ & $210 \mathrm{~W}$ & $67 \mathrm{~N}$ & $67 \mathrm{~N}$ & $74 \mathrm{~V}$ & $103 \mathrm{~N}$ & & & & 1035 & 751 & $67 \mathrm{~N}$ & $101 \mathrm{Q}$ & $67 \mathrm{G}$ & 2151 & 2151 & $70 \mathrm{R}$ \\
\hline & $98 \mathrm{G}$ & $188 \mathrm{~L}$ & $215 Y$ & 751 & $70 R$ & $190 \mathrm{~A}$ & 751 & $101 \mathrm{Q}$ & 101E & & $101 \mathrm{Q}$ & 1081 & $215 Y$ & $75 \mathrm{~L}$ & $70 R$ & $98 \mathrm{G}$ & 1181 & & & & $184 \mathrm{~V}$ & 1181 & $75 \mathrm{M}$ & $103 \mathrm{~N}$ & $103 \mathrm{~N}$ & $219 Q$ & $219 Q$ & $184 \mathrm{~V}$ \\
\hline & $103 N$ & $215 Y$ & & $103 \mathrm{~N}$ & $74 \mathrm{~V}$ & $184 \mathrm{~V}$ & $103 \mathrm{~N}$ & $184 \mathrm{~V}$ & $103 \mathrm{~N}$ & & $181 C$ & $115 \mathrm{~F}$ & $219 Q$ & 1181 & $190 \mathrm{~A}$ & 1181 & $184 \mathrm{~V}$ & & & & $190 \mathrm{~A}$ & $210 \mathrm{~W}$ & $101 \mathrm{Q}$ & 1081 & 1181 & & & \\
\hline & 1181 & & & 1081 & $103 \mathrm{~N}$ & $219 Q$ & 1081 & $215 Y$ & 1181 & & $190 \mathrm{~S}$ & $151 \mathrm{M}$ & & $181 \mathrm{C}$ & $227 \mathrm{~L}$ & $184 \mathrm{~V}$ & $210 \mathrm{~W}$ & & & & $215 \mathrm{~F}$ & $215 \mathrm{~F}$ & 1181 & $181 \mathrm{C}$ & $184 \mathrm{~V}$ & & & \\
\hline & $210 \mathrm{~F}$ & & & 1181 & $181 C$ & & 1181 & & $184 \mathrm{~V}$ & & $215 Y$ & $179 \mathrm{E}$ & & $184 \mathrm{~V}$ & $184 \mathrm{~V}$ & $210 \mathrm{~W}$ & $215 Y$ & & & & $219 \mathrm{Q}$ & & $184 \mathrm{~V}$ & $190 \mathrm{~A}$ & $210 \mathrm{~W}$ & & & \\
\hline & $215 Y$ & & & $210 \mathrm{~W}$ & $210 \mathrm{~W}$ & & $178 \mathrm{M}$ & & $210 \mathrm{~W}$ & & & $184 \mathrm{~V}$ & & $190 \mathrm{~A}$ & $210 \mathrm{~W}$ & $215 Y$ & & & & & & & $210 \mathrm{~W}$ & $210 \mathrm{~W}$ & $215 \mathrm{~F}$ & & & \\
\hline & $219 R$ & & & $215 Y$ & $215 \mathrm{~F}$ & & $210 \mathrm{~W}$ & & $215 Y$ & & & $219 \mathrm{E}$ & & $210 \mathrm{~S}$ & $215 \mathrm{~F}$ & $227 \mathrm{~L}$ & & & & & & & $215 \mathrm{~F}$ & $215 Y$ & & & & \\
\hline & & & & & $219 \mathrm{Q}$ & & $215 Y$ & & $219 \mathrm{E}$ & & & & & $215 Y$ & $219 R$ & & & & & & & & & & & & & \\
\hline Protease & $10 \mathrm{~F}$ & $10 \mathrm{~V}$ & $10 \mathrm{~V}$ & 101 & $10 \mathrm{~V}$ & $10 \mathrm{~F}$ & 101 & 101 & 101 & 101 & $10 \mathrm{~V}$ & 101 & 20R & 101 & $10 \mathrm{~V}$ & $10 \mathrm{~F}$ & $10 \mathrm{~F}$ & 101 & $10 \mathrm{~F}$ & 101 & 101 & 101 & 101 & $10 \mathrm{~F}$ & $10 R$ & $10 \mathrm{~F}$ & $10 \mathrm{~V}$ & 201 \\
\hline \multirow{8}{*}{ mutations } & 201 & 461 & $20 \mathrm{R}$ & $33 \mathrm{~F}$ & $20 \mathrm{R}$ & $20 \mathrm{R}$ & $33 \mathrm{~F}$ & $53 \mathrm{~L}$ & $33 \mathrm{~F}$ & $33 \mathrm{~V}$ & 46L & 201 & 321 & 241 & 201 & $33 \mathrm{~F}$ & 461 & $20 \mathrm{R}$ & $33 \mathrm{~F}$ & $20 \mathrm{R}$ & 20R & 461 & $13 \mathrm{~V}$ & 461 & 321 & 201 & $13 \mathrm{~V}$ & 361 \\
\hline & 361 & $47 \mathrm{~V}$ & 361 & $46 \mathrm{~L}$ & $33 \mathrm{~F}$ & 461 & $36 \mathrm{~L}$ & $54 \mathrm{~V}$ & $46 \mathrm{~L}$ & $60 \mathrm{E}$ & $54 \mathrm{~V}$ & 361 & 461 & $33 \mathrm{~F}$ & 361 & 46L & $54 \mathrm{M}$ & 241 & $54 \mathrm{~V}$ & $35 \mathrm{D}$ & 361 & $47 \mathrm{~V}$ & 321 & $47 \mathrm{~V}$ & $33 \mathrm{~F}$ & 361 & 241 & $54 \mathrm{~V}$ \\
\hline & 461 & $71 \mathrm{~V}$ & 461 & $76 \mathrm{~V}$ & 361 & $54 \mathrm{~V}$ & $46 \mathrm{~L}$ & $71 \mathrm{~V}$ & $54 \mathrm{~V}$ & $76 \mathrm{~V}$ & $63 \mathrm{P}$ & 461 & $76 \mathrm{~V}$ & 461 & 461 & 54L & $71 \mathrm{~V}$ & 361 & $71 \mathrm{~V}$ & 461 & 461 & $71 \mathrm{~V}$ & $33 \mathrm{~F}$ & $76 \mathrm{~V}$ & 461 & 461 & $33 \mathrm{~F}$ & $76 \mathrm{~V}$ \\
\hline & $50 \mathrm{~V}$ & $76 \mathrm{~V}$ & $76 \mathrm{~V}$ & $82 \mathrm{~F}$ & $54 \mathrm{~V}$ & $63 \mathrm{P}$ & $76 \mathrm{~V}$ & $76 \mathrm{~V}$ & 711 & & $71 \mathrm{~V}$ & $54 \mathrm{~L}$ & $82 \mathrm{~A}$ & $54 \mathrm{~V}$ & $47 \mathrm{~V}$ & $71 \mathrm{~V}$ & $76 \mathrm{~V}$ & 461 & $76 \mathrm{~V}$ & $54 \mathrm{~V}$ & $54 \mathrm{~V}$ & $76 \mathrm{~V}$ & 361 & $84 \mathrm{~V}$ & $47 \mathrm{~V}$ & $76 \mathrm{~V}$ & 461 & $82 \mathrm{~A}$ \\
\hline & $541 \mathrm{~N}$ & 771 & $90 \mathrm{M}$ & $90 \mathrm{M}$ & 735 & $76 \mathrm{~V}$ & $82 \mathrm{~F}$ & 771 & $76 \mathrm{~V}$ & & $82 \mathrm{~A}$ & $76 \mathrm{~V}$ & & $63 \mathrm{P}$ & $53 \mathrm{~L}$ & $76 \mathrm{~V}$ & $82 \mathrm{~A}$ & $54 \mathrm{~V}$ & 771 & $76 \mathrm{~V}$ & $71 \mathrm{~V}$ & $90 \mathrm{M}$ & 461 & & $76 \mathrm{~V}$ & $84 \mathrm{~V}$ & $54 \mathrm{~V}$ & \\
\hline & $76 \mathrm{~V}$ & & & & $76 \mathrm{~V}$ & $82 \mathrm{~F}$ & $84 \mathrm{~V}$ & $82 \mathrm{~A}$ & $77 \mathrm{~L}$ & & $93 \mathrm{~L}$ & $84 \mathrm{~V}$ & & $71 \mathrm{~V}$ & $76 \mathrm{~V}$ & 771 & & $76 \mathrm{~V}$ & $82 \mathrm{~A}$ & & $76 \mathrm{~V}$ & & $76 \mathrm{~V}$ & & $84 \mathrm{~V}$ & & $76 \mathrm{~V}$ & \\
\hline & & & & & $90 \mathrm{M}$ & & $90 \mathrm{M}$ & $84 \mathrm{~V}$ & $82 \mathrm{~A}$ & & & & & $76 \mathrm{~V}$ & $84 \mathrm{~V}$ & $84 \mathrm{~V}$ & & $82 \mathrm{C}$ & & & $82 \mathrm{~F}$ & & $84 \mathrm{~V}$ & & 885 & & $82 \mathrm{~A}$ & \\
\hline & & & & & & & & $90 \mathrm{M}$ & $90 \mathrm{M}$ & & & & & $82 \mathrm{~A}$ & $90 \mathrm{M}$ & $90 \mathrm{M}$ & & & & & $90 \mathrm{M}$ & & $90 \mathrm{M}$ & & $90 \mathrm{M}$ & & & \\
\hline
\end{tabular}

Active Drug

Scores

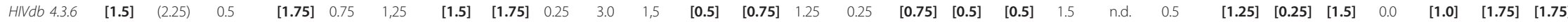
$\begin{array}{llllllllllllllllllllllllllllllll}\text { Rega V7.1.1 } & 2.0 & (2.75) & 1.5 & 2.0 & 1.0 & 1.0 & 2.0 & 3.5 & 0.0 & 3.0 & 1.75 & {[1.0]} & {[1.5]} & 1.0 & 0.0 & {[0.25]} & {[0.75]} & {[0.75]} & 1.75 & \text { n.d. } & 0.75 & {[1.0]} & {[0.5]} & 2.75 & 0.5 & 2.0 & 2.25 & 4.25\end{array}$

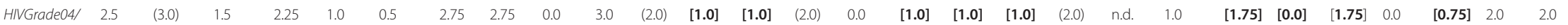
$\begin{array}{llllllllllllllllllllllllllllllll}\text { ANRS 10/ } & 3.0 & (2.0) & 0.5 & {[1.0]} & 1.0 & 1.0 & 2.0 & 2.5 & 0.5 & 3.0 & 1.5 & {[0.0]} & 2.0 & 1.0 & 0.5 & {[1.0]} & {[1.0]} & {[0.5]} & (2.0) & \text { n.d. } & 0.5 & 2.0 & {[0.5]} & 2.5 & 1.5 & {[1.5]} & 2.5 & 3.0 & 0\end{array}$ 2007 $\begin{array}{lllllllllllllllllllllllllllll}\text { geno2pheno } & 2.0 & (3.0) & 1.0 & 2.5 & (2.0) & \text { n.d. } & \text { n.d. } & 2.0 & \text { n.d. } & 3.0 & (2.0) & {[0.5]} & {[0.5]} & 1.5 & 0.0 & {[1.0]} & \text { n.d. } & \text { n.d. } & \text { n.d. } & \text { n.d. } & 0.0 & 2.5 & \text { n.d. } & 2.0 & 0.0 & 1.0 & 2.0 & 4.0\end{array}$

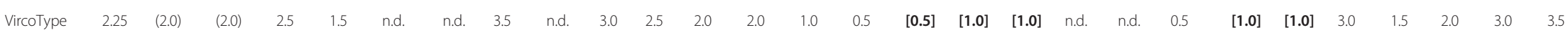

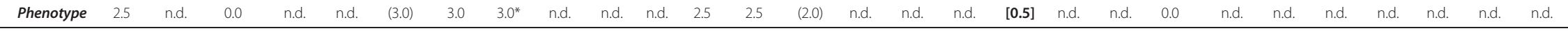
Follow-up

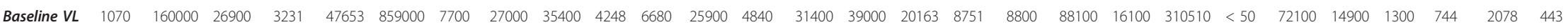

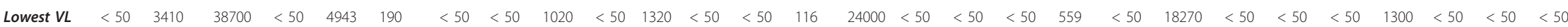
(12-96

weeks

$\begin{array}{lllllllllllllllllllllllllllll}\text { VL at week } & 13589 & 46570 & 152000 & <50 & 20626 & \text { LFU } & \text { LFU } & \text { LFU } & 16500 & \text { LFU } & \text { LFU } & <50 & <50 & 116 & \text { LFU } & <50 & <50 & \text { LFU } & \text { LFU } & 1777 & \text { LFU } & 391 & 60 & <50 & \text { LFU } & <50 & <50 & <50\end{array}$ 96

Based on five genotypic resistance interpretation tools as well as two phenotypic resistance tests, an active drug score (ADS) for the follow-up therapy was calculated by adding the activity score for every active drug ranging from $\mathrm{AS}=1.0$ for complete sensitivity, $\mathrm{AS}=0.75$ for potential low-level resistance, $\mathrm{AS}=0.5$ for intermediate resistance, $\mathrm{AS}=0.25$ for possible resistance and $\mathrm{AS}=0.0$ for complete resistance. Their prediction on follow-up therapy was then compared with the virological response in a time frame of 96 weeks. Sucessfull therapies despite an active drug score prediction of $<2$ are displayed in bold and square brackets. Unsucessfull therapies despite an active drug score prediction of $\geq 2$ are displayed in round brackets. LFU=loss of follow-up. 
Table 2 Comparison of results (group A and group B)

\begin{tabular}{|c|c|c|c|c|c|c|}
\hline & \multicolumn{3}{|c|}{ Virus Suppression } & \multicolumn{3}{|c|}{ Therapy success } \\
\hline & $\begin{array}{c}\text { Group A } \\
\text { Median } \\
\text { Viral load }\end{array}$ & $\begin{array}{c}\text { Group B } \\
\text { Median Viral load }\end{array}$ & Mann-Whitney & $\begin{array}{l}\text { Group A } \\
\text { Success }\end{array}$ & Group B Success & $\chi^{2}$ \\
\hline Baseline & $\begin{array}{c}26,950 \\
(N=10)\end{array}$ & $\begin{array}{c}8,800 \\
(N=17)\end{array}$ & $P=0.12$ & $\begin{array}{c}0 \% \\
(N=10)\end{array}$ & $\begin{array}{c}5.9 \% \\
(N=17)\end{array}$ & $P=0.998$ \\
\hline Week 12 & $\begin{array}{c}370 \\
(N=10)\end{array}$ & $\begin{array}{c}<50 \\
(N=15)\end{array}$ & $P=0.07$ & $\begin{array}{c}40.0 \% \\
(N=10)\end{array}$ & $\begin{array}{c}66.7 \% \\
(N=15)\end{array}$ & $P=0.035$ \\
\hline Week 24 & $\begin{array}{c}4650 \\
(N=8)\end{array}$ & $\begin{array}{c}<50 \\
(N=13)\end{array}$ & $P=0.16$ & $\begin{array}{l}37.5 \% \\
(\mathrm{~N}=8)\end{array}$ & $\begin{array}{c}69.2 \% \\
(\mathrm{~N}=13) \\
\end{array}$ & $P=0.166$ \\
\hline Week 48 & $\begin{array}{c}3410 \\
(\mathrm{~N}=7)\end{array}$ & $\begin{array}{c}<50 \\
(N=13) \\
\end{array}$ & $P=0.19$ & $\begin{array}{l}42.9 \% \\
(\mathrm{~N}=7)\end{array}$ & $\begin{array}{c}53.8 \% \\
(N=13)\end{array}$ & $P=0.425$ \\
\hline Week 96 & $\begin{array}{l}15,045 \\
(N=6)\end{array}$ & $\begin{array}{l}<50 \\
(N=9)\end{array}$ & $P=0.044$ & $\begin{array}{l}16.7 \% \\
(N=6)\end{array}$ & $\begin{array}{l}54.5 \% \\
(\mathrm{~N}=9)\end{array}$ & $P=0.002$ \\
\hline
\end{tabular}

Comparison of results (group A and group B). Median viral loads and the therapy success rates illustrate a significantly better long-term suppression of HIV when SQV/ATV plus optimized backbone therapy is combined with a L76V-selecting drug. In bivariate analysis, these results were independent from slightly different baseline viral loads $(<0,5 \mathrm{log})$ between group $A$ and $B$.

therapy failure were diagnosed positive for L90 M. The remaining two were therapy non-compliant. Thus, it might be questionable if SQV, which primarily selects L90 M should be replaced in favour of ATV [18-20]. In addition, due to the approval of new drug classes over the past years one might err on the side of caution to supplement therapy regimens with new drugs. Due to the low potency of the present cohort and varying amounts of HIV non-B infected individuals in the examined patient groups, caution should be additionally advised, since these limitations might have effect on clinical outcomes.

Nevertheless, since there are still distinct discrepancies, mostly overestimation of resistance, in the prediction of the resistance level for Atazanavir and Saquinavir in five of the most common genotypic interpretation systems, there is still a need for further evaluation in the case of L76V occurrence.

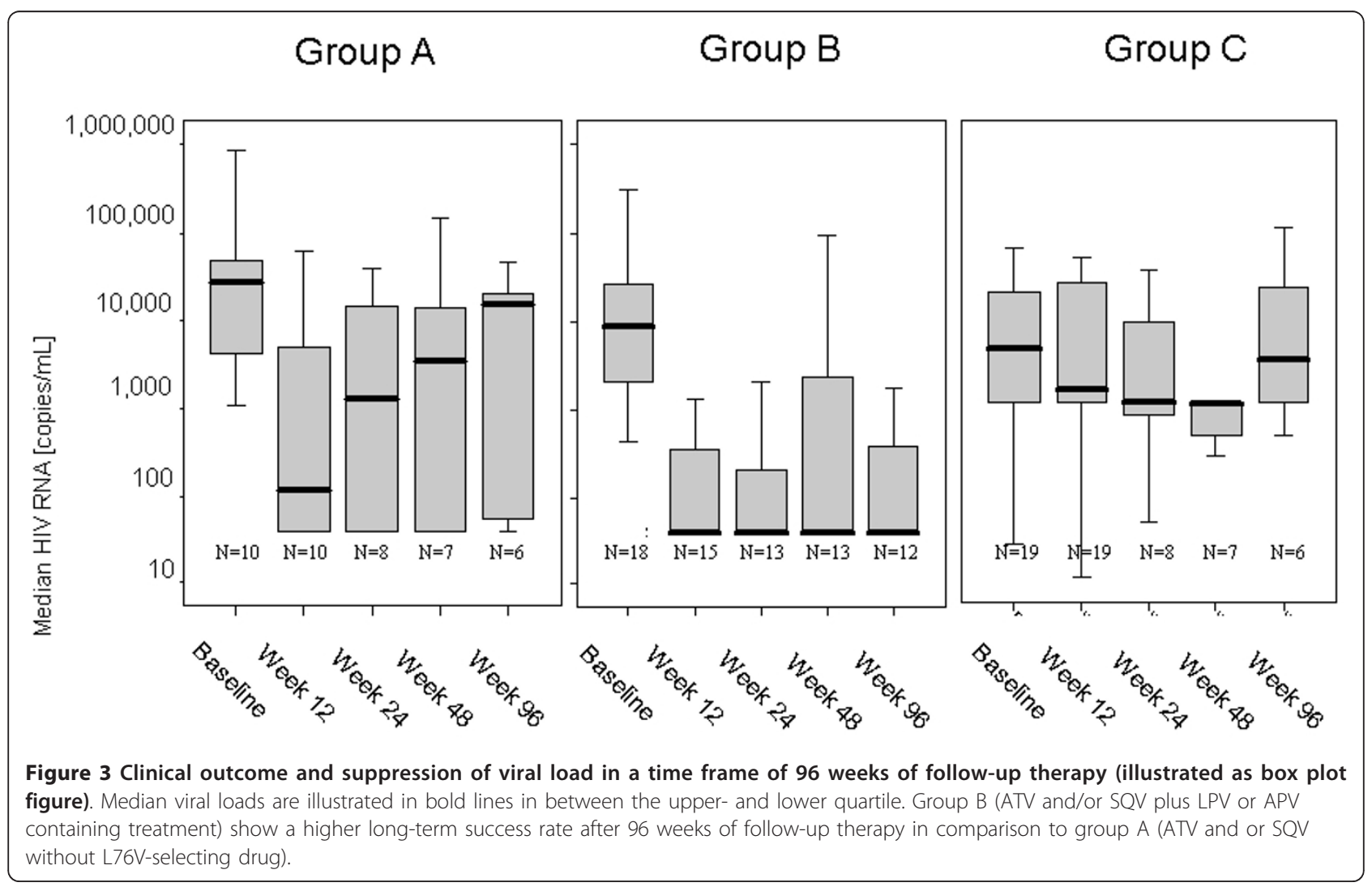


Table 3 Compensatory changes in virus genotypes within 96 weeks of follow-up therapy

\begin{tabular}{|c|c|c|c|c|c|}
\hline Group & $\begin{array}{l}\text { Patient } \\
\text { ID }\end{array}$ & Protease mutations at start of therapy & $\begin{array}{l}\text { Time of } \\
\text { therapy } \\
\text { failure }\end{array}$ & $\begin{array}{l}\text { Time of } 2 \mathrm{nd} \\
\text { genotype }\end{array}$ & Protease mutations after therapy failure \\
\hline \multirow[t]{4}{*}{ A } & $\# 1$ & $\begin{array}{l}\text { L10FL, K20I, M36I, M46I, I50V, I54IV, L63P, } \\
\text { L76V }\end{array}$ & Week 48 & Week 144 & 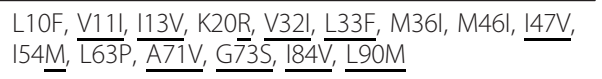 \\
\hline & $\# 2$ & L10V, M46I, 147V, L63P, A71V, L76V, V77I & Week 12 & Week 48 & $\begin{array}{l}\mathrm{L} 63 \mathrm{P}, \mathrm{V} 77 \mathrm{I} \\
\text { (therapy interruption) }\end{array}$ \\
\hline & $\# 4$ & L10V, K20RK, M36I, M46I, L63P, L76V, L90M & Week 12 & Week 24 & L10V, K20R, M361, M46I, F53L, L63P, I84IV, L90M \\
\hline & $\# 22$ & $\begin{array}{l}\text { L10I, L33F, M46L, I54V, L63P, A71I, L76V, } \\
\text { V77I, V82A, L90M }\end{array}$ & Week 12 & Week 48 & $\begin{array}{l}\text { L10I, L33F, M46L, F53L, I54V, L63P, A71T, G73S, V77I, } \\
\text { V82A, L90M }\end{array}$ \\
\hline \multirow[t]{4}{*}{ B } & $\# 8$ & K20R, V32I, M46I, L76V, V82A & Week 48 & Week 48 & K20R, V32I, M36I, M46I, F53FL, L76V, V82A, L90LM \\
\hline & $\# 9$ & $\begin{array}{l}\text { L10I, L24I, L33F, M46I, I54V, L63P, A71V, } \\
\text { L76V, V82A }\end{array}$ & Week 12 & Week 24 & $\begin{array}{l}\text { L10I, L24I, L33F, M46I, F53L, 154V, L63P, A71V, L76V, } \\
\text { V82A, I84V }\end{array}$ \\
\hline & $\# 10$ & $\begin{array}{l}\text { L10IV, K20I, M36I, M46I, I47V, F53L, L63P, } \\
\text { A71V, G73 D, L76VV, I84V, L90M }\end{array}$ & $\begin{array}{l}\text { Week } 12 \\
\text { compliance }\end{array}$ & Week 48 & $\begin{array}{l}\text { L10V, K20I, L33I, M361, M46I, I47V, F53L, L63P, A71V, } \\
\text { G73 D, L76V, I84V, L90M }\end{array}$ \\
\hline & \#27 & L10I, M46I, I47V, L63P, A71V, L76V, L90M & Week 48 & Week 48 & L10I, M46I, I47V, L63P, A71V, L76V, I84V, L90M \\
\hline \multirow[t]{2}{*}{$C$} & $\# 6$ & $\begin{array}{l}\text { L10V, L33F, M46L, 154V, A71V, L63P, A71V, } \\
\text { L76V, V82A }\end{array}$ & Week 12 & Week 96 & $\begin{array}{l}\text { L10V, K20R, L33F, M361, M46L, I54V, A71V, L76V, } \\
\text { V82A }\end{array}$ \\
\hline & \#23 & $\begin{array}{l}\text { L10FIRV, L33F, I54MV, D60E, L63P, A71V, } \\
\text { L76V, V82F }\end{array}$ & Week 12 & Week 24 & $\begin{array}{l}\text { L10FIRV, L33F, I54MV, D60E, L63P, A71TNN, L76V, } \\
\text { V82F }\end{array}$ \\
\hline
\end{tabular}

Compensatory changes in virus genotypes within 96 weeks of follow-up therapy. Patients with failing therapies within the 96 weeks received a second resistance testing. While L76V was still present in patients receiving L76V-selecting drugs, it was then absent in patients without these drugs (new detected mutation are underlined). Therapy failure in group B was noticeable associated with an additional establishment of the protease mutation L90 M.

\section{Methods}

\section{Clinical material}

HIV strains of 46 intensely pretreated (34 showed NRTI/NNRTI/PI resistance/12 showed NRTI/PI resistance) and one naïve with transmitted mutation, L76Vpositive patients derived from 24 centres throughout Germany between 1999-2009 were retrospectively analysed for HIV-resistance patterns and success of follow-up therapy. The inclusion criterion is provided in Figure 4. Descriptional statistics concerning person-toperson variations of virological and immunological parameters were assessed at baseline before switch of therapy and are provided in Table 4.

All patient data was categorized in three groups concerning the follow-up therapy:

Group A: ATV and/or SQV (no selection pressure on L76V)

Group B: LPV or APV plus ATV or SQV (maintained selection pressure on L76V)

Group C: LPV or APV plus other drugs (maintained selection pressure on L76V)

All patients received an optimized backbone therapy.

\section{HIV-1 RNA Quantification}

Plasma of patients was analysed at baseline and week 12, 24, 48 until end of investigation at week 96 to monitor efficiency of therapy. Plasma RNA was measured by using the COBAS AMPLICOR HIV-1 Monitor system and the Abbott m2000sp/rt system according to the manufacturer's recommendations.

\section{Genotypic resistance testing}

Plasma samples of all 47 patients were collected and stored at $-20^{\circ} \mathrm{C}$ until time of RNA extraction. All specimens were processed by using the FDA-approved Siemens HIV TruGene system as well as the Abbott HIV-1 Genotyping System on the Applied Biosystems' 3100 capillary electrophoresis platform according to the manufacturer's recommendations. HIV-1 genotypes were processed and analyzed by using the wildtype LAV-1 sequence as reference. The sensitivity for detecting minor quasispecies variants was $15 \%$. In this cohort, only patients with major $\mathrm{L} 76 \mathrm{~V}$ positive population were included. Minor wildtype variants were not detected at this position.

\section{Phenotypic resistance testing}

Phenotypic resistance analysis of the complete protease gene and the first 900bp of the RT were performed according to an earlier described recombinant virus assay by determining a virus specific resistance factor [21]. In addition, the Virtual Phenotype ${ }^{\mathrm{TM}}$ (based on 53,000 paired genotypes and phenotypes) from Virco was assessed for those patient samples where no recombinant virus assay was realizable.

\section{Interpretation of drug resistance}

Several algorithms are available worldwide, both in public and private domains. The concordance of resistance predictions was analysed between the five most commonly used algorithms [REGA v7.1.1 [22] HIVGrade 


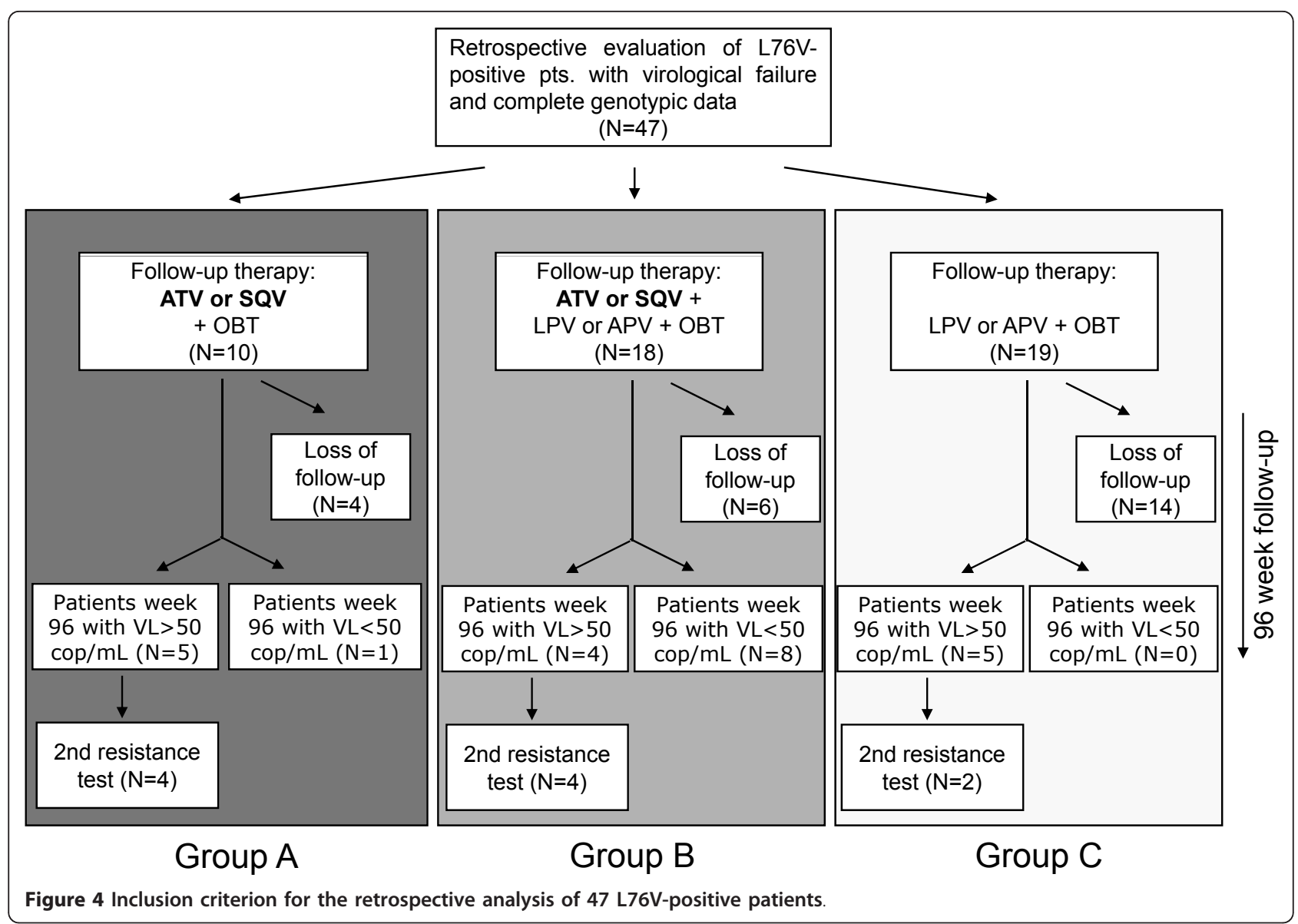

ver.12/2008 [23] ANRS ver.10/2007 [24] Stanford HIVdb ver.4.3.6 [25] and the geno2pheno online tool [26]] for the drugs ATV, SQV, LPV and/or APV. Multiple resistance tests in treatment history were cumulatively documented. In addition, an active drug score (ADS) was determined in order to analyze the amount of remaining active drugs in follow-up therapies of each patient (susceptible $=+1 /$ low intermediate $=+0.75 /$ intermediate $=$ $+0.5 /$ high intermediate $=+0.25 /$ resistant $=+0.0)$. This ADS allowed a statement concerning the prediction of

Table 4 Patient characteristics and parameters

\begin{tabular}{|c|c|c|c|c|}
\hline Parameter & Total & Group A ( $N=10)$ & Group B ( $N=18)$ & Group $C(N=19)$ \\
\hline \multicolumn{5}{|l|}{ Gender } \\
\hline Male & $84 \%$ & $100 \%$ & $100 \%$ & $68,7 \%$ \\
\hline Female & $16 \%$ & $0 \%$ & $0 \%$ & $31,3 \%$ \\
\hline \multicolumn{5}{|l|}{ HIV-1 subtype } \\
\hline Patients with subtype B & $69 \%$ & $80 \%$ & $64 \%$ & $67 \%$ \\
\hline Patients with non-B subtype & $31 \%$ & $20 \%$ & $36 \%$ & $33 \%$ \\
\hline \multicolumn{5}{|l|}{ Treatment history } \\
\hline Mean duration under ART in months (mean) & 66 & 80 & 88 & 54 \\
\hline Current active drug score (mean) & - & 1.5 & 1.3 & 0.5 \\
\hline \multicolumn{5}{|l|}{ HIV-1 RNA [copies/ml; median] } \\
\hline Baseline & 20,163 & 26,950 & 8,800 & 42,600 \\
\hline \multicolumn{5}{|l|}{ CD4 cell counts [cells/ $\mu$; median] } \\
\hline Baseline & 260 & 291 & 307 & 246 \\
\hline
\end{tabular}

Patient characteristics and parameters. All patients were categorized in three groups as described throughout the article: Group A (ATV and or SQV), group B (ATV and or SQV plus L76V selecting drug LPV, APV or DRV) and group C (L76V selecting drug plus optimized backbone therapy). 
follow-up therapy. It is generally accepted that a successful therapy should contain at least two active drugs, preferably three (ADS $\geq 2.0)[27,28]$.

\section{Author details}

'PZB Aachen, HIV\&Hepatitis Research Group, Blondelstr., 52062 Aachen, Germany. ${ }^{2}$ University of Erlangen, Institute for Clinical and Molecular Virology, Schloßgarten, D-91504 Erlangen, Germany. ${ }^{3}$ University of Cologne, Institute for Virology, Fürst Pückler Str. 56, D-50925 Cologne, Germany. ${ }^{4}$ University of Frankfurt, Institute for Virology, Paul-Ehrlich-Str. 40, D-60596 Frankfurt, Germany. ${ }^{5}$ Roche Pharma, Clinical Project Management, Emil-BarellStr. 1, D-79639 Grenzach-Wyhlen, Germany. ${ }^{6}$ Laboratories Thiele, Institute for Immunology and Genetics, Hellmut-Hartert-Str. 1, D-67655 Kaiserslautern, Germany. ${ }^{7}$ Medical Laboratories Berg, HIV Research, Seestr. 13, D-13353 Berlin, Germany.

\section{Authors' contributions}

FW has made substantive intellectual contribution to the study including acquisition-, analysis- and interpretation of data and finally drafting the manuscript. JV assisted as consultant in patient-specific aspects and was involved in manuscript revision. GN was responsible with genotyping processes as described in the manuscript. RE was responsible for genotypic resistance interpretation and manuscript revision. HW realized the phenotypic resistance analysis. PB and AT assissted in concept and design aspects and directed sample- and data acquisition. HK, RK, MS and TB were significantly involved in data acquisition, provision of samples and manuscript revision. All authors read and approved the final manuscript.

\section{Competing interests}

The authors declare that they have no competing interests.

Received: 27 September 2010 Accepted: 13 February 2011 Published: 13 February 2011

\section{References}

1. Cong ME, Heneine W, Garcia-Lerma JG: Mutational interactions modulate the fitness cost of drug-resistant HIV-1. J Virol 2007, 81(6):3037-41.

2. Hu Z, Giguel F, Hatano H, Reid P, Lu J, Kuritzkes DR: Fitness comparison of thymidine analog resistance pathways in human immunodeficiency virus type 1. J Virol 2006, 80(14):7020-7.

3. Catucci M, Venturi G, Romano L, Riccio ML, De Milito A, Valensin PE, Zazzi M: Development and significance of the HIV-1 reverse transcriptase M184V mutation during combination therapy with lamivudine, zidovudine, and protease inhibitors. J Acquir Immune Defic Syndr 1999, 21(3):203-8.

4. Masquelier B, Descamps D, Carriere I, Ferchal F, Collin G, Denayrolles M, Ruffault A, Chanzy B, Izopet J, Buffet-Janvresse C, Schmitt MP, Race E, Fleury HJ, Aboulker JP, Yeni P, Brun-Vézinet F: Zidovudine resensitization and dual HIV-1 resistance to zidovudine and lamivudine in the delta lamivudine roll-over study. Antivir Ther 1999, 4(2):69-77.

5. Matamoros T, Franco S, Vazquez-Alvarez BM, Mas A, Martinez MA, MendezArias L: Molecular determinants of multi-nucleoside analogue resistance in HIV-1 reverse transcriptases containing a dipeptide insertion in the fingers subdomain: effect of mutations D67N and T215Y on removal of thymidine nucleotide analogues from blocked DNA primers. J Biol Chem 2004, 279(23):24569-77.

6. Gallant JE: The M184V mutation: what it does, how to prevent it, and what to do with it when it's there. AIDS Read 2006, 16(10):556-9.

7. Wolf $\mathrm{K}$, Walter $\mathrm{H}$, Beerenwinkel $\mathrm{N}$, Keulen W, Kaiser R, Hoffmann D, Lengauer T, Selbig J, Vandamme AM, Korn K, Schmidt B: Tenofovir Resistance and Resensitization. Antimicrob Agents Chemother 2003, 47(11):3478-84.

8. Götte M, Weinberg MA: Biochemical mechanisms involved in overcoming HIV resistance to nucleoside inhibitors of reverse transcriptase. Drug Resist Updat 2000, 3(1):30-38.

9. de Mendoza C, Valer L, Bacheler L, Pattery T, Corral A, Soriano V: Prevalence of the HIV-1 protease mutation I47A in clinical practice and association with lopinavir resistance. AIDS 2006, 20(7):1071-4.
10. Ziermann R, Limoli K, Das K, Arnold E, Petropoulos J, Parkin NT: A mutation in human immunodeficiency virus type 1 protease, N88 S, that causes in vitro hypersusceptibility to amprenavir. J Virol 74:4414-4419.

11. Andreoni M: Viral phenotype and fitness. New Microbiol 2004, 27(2 Suppl 1):71-6.

12. Svarovskaia ES, Feng JY, Margot NA, Myrick F, Goodman D, Ly JK, White KL, Kutty N, Wang R, Borroto-Esoda K, Miller MD: The A62V and S68G mutations in HIV-1 reverse transcriptase partially restore the replication defect associated with the K65R mutation. J Acquir Immune Defic Syndr 2008, 48(4):428-36.

13. Averbuch D, Schapiro JM, Lanier ER, Gradstein S, Gottesman G, Kedem E, Einhorn M, Grisaru-Soen G, Ofir M, Engelhard D, Grossman Z: Diminished selection for thymidine-analog mutations associated with the presence of M184V in Ethiopian children infected with HIV subtype C receiving lamivudine-containing therapy. Pediatr Infect Dis J 2006, 25(11):1049-56.

14. Nijhuis $M$, Wensing AM, Bierman WF, de Jong D, Kagan R, Fun A, Jaspers CA, Schurink KA, van Agtmael MA, Boucher CA: Failure of Treatment with First-Line Lopinavir Boosted with Ritonavir Can Be Explained by Novel Resistance Pathways with Protease Mutations 76V. J Infect Dis 2009, 200(5):698-709.

15. Alcaro S, Artese A, Ceccherini-Silberstein F, Ortuso F, Perno CF, Sing T, Svicher V: Molecular Dynamics and Free Energy Studies on the WildType and Mutated HIV-1 Protease Complexed with Four Approved Drugs: Mechanism of Binding and Drug Resistance. J Chem Inf Model 2009, 49(7):, 1751-1761.

16. Zaccarelli M, Tozzi V, Perno CF, Antinori A: The challenge of antiretroviraldrug-resistant HIV: is there any possible clinical advantage? Curr HIV Res 2004, 2(3):283-92.

17. Rhee SY, Taylor J, Wadhera G, Ben-Hur A, Brutlag DL, Shafer RW: Genotypic predictors of human immunodeficiency virus type 1 drug resistance. Proc Natl Acad Sci USA 2006, 103:17355-17360.

18. Zolopa AR, Shafer RW, Warford A, Montoya JG, Hsu P, Katzenstein D, Merigan TC, Efron B: HIV-1 genotypic resistance patterns predict response to saquinavir-ritonavir therapy in patients in whom previous protease inhibitor therapy had failed. Ann Intern Med 1999, 131:813-821.

19. Dragsted UB, Gerstoft J, Youle M, Fox Z, Losso M, Benetucci J, Jayaweera DT, Rieger A, Bruun JN, Castagna A, Gazzard B, Walmsley S, Hill A, Lundgren JD: A randomized trial to evaluate lopinavir/ritonavir versus saquinavir/ritonavir in HIV-1-infected patients: the MaxCmin2 trial. Antivir Ther 2005, 10:735-743.

20. Clotet B, Bellos N, Molina JM, Cooper D, Goffard JC, Lazzarin A, Wohrmann A, Katlama C, Wilkin T, Haubrich R, Cohen C, Farthing C, Jayaweera D, Markowitz M, Ruane P, Spinosa-Guzman S, Lefebvre E: Efficacy and safety of darunavir-ritonavir at week 48 in treatment-experienced patients with HIV-1 infection in POWER 1 and 2: a pooled subgroup analysis of data from two randomised trials. Lancet 2007, 369:1169-1178.

21. Walter H, Schmidt B, Korn K, Vandamme AM, Harrer T, Uberla K: Rapid, phenotypic HIV-1 drug sensitivity assay for protease and reverse transcriptase inhibitors. J Clin Virol 1999, 13(1-2):71-80.

22. REGAv.7.1.1. [http://bioafrica.net/rega-genotype/html/subtypinghiv.html].

23. HIV-Grade ver 12/2008. [http://www.hiv-grade.de].

24. ANRS ver. 10/2007 Agence Nationale de Recherche sur le SIDA (ANRS). [http://www.hivfrenchresistance.org].

25. Stanford HIVdb ver.4.3.6. [http://hivdb.stanford.edu].

26. geno2pheno. [http://www.geno2pheno.org].

27. Raltegravir Treatment in Patients Failing Highly Active Antiretroviral Therapy (HAART) in Denmark. [http://clinicaltrials.gov/ct2/show/ NCT01061957]

28. Thompson MA, Aberg JA, Cahn P, Julio S, Montaner G, Rizzardini G, Telenti A, Gatell JM, Günthard HF, Hammer SM, Hirsch MS, Jacobsen DM, Reiss P, Richman DD, Volberding PA, Yeni P, Schooley RT, International AIDS Society-USA: Antiretroviral Treatment of Adult HIV Infection - 2010 Recommendations of the International AIDS Society-USA Panel. JAMA 2010, 304(3):321-333.

doi:10.1186/1742-6405-8-7

Cite this article as: Wiesmann et al:: The L76V mutation in HIV-1 protease is potentially associated with hypersusceptibility to protease inhibitors Atazanavir and Saquinavir: is there a clinical advantage? AIDS Research and Therapy 2011 8:7. 